\title{
Caracterização Mecânica e Análise Microestrutural de Chapas Obtidas pelo Processo de Tailor Welded Blank (TWB)
}

\author{
Etiene Pereira de Andrade ${ }^{1}$ (D), Guilherme Souza Assunção ${ }^{1}$ (D), Wellington Augusto dos Santos ${ }^{2}$, Ivanilza Felizardo ${ }^{3}$, \\ Alexandre Queiroz Bracarense ${ }^{1}$ (D) \\ ${ }^{1}$ Universidade Federal de Minas Gerais - UFMG, Belo Horizonte, MG, Brasil. \\ 2 FIAT Chrysler Automóveis do Brasil LTDA, Betim, MG, Brasil. \\ ${ }^{3}$ Centro Federal de Educação Tecnológica de Minas Gerais - CEFET-MG, Belo Horizonte, MG, Brasil.
}

Como citar: Andrade EP, Assunção GS, Santos WA, Felizardo I, Bracarense AQ. Caracterização mecânica e análise microestrutural de chapas obtidas pelo processo de Tailor Welded Blank (TWB). Soldagem \& Inspeção. 2019;24:e2425. https://doi.org/10.1590/0104-9224/SI24.25

\begin{abstract}
Resumo: Um dos maiores desafios da indústria automobilística, a nível global, trata-se de desenvolver veículos que sejam, simultaneamente, leves e resistentes mecanicamente. O Tailor Welded Blank (TWB) surge como solução, pois é um processo que permite a soldagem de topo de chapas de diferentes especificações (material, espessura e/ou revestimento), antes da conformação. Nesta pesquisa trabalhou-se com chapas de aços livre de intersticiais (IF - Intertitial Free), da Companhia Siderúrgica Nacional (CSN), de códigos FEE 210 de 1,2 mm de espessura e IF CSN FEP 05 de $0,7 \mathrm{~mm}$ de espessura, soldadas a laser. Foram feitos ensaios de tração uniaxial e de microdureza Vickers. A linha de solda foi orientada de $0^{\circ}$ a $70^{\circ}$ (variando de $5^{\circ}$ em $5^{\circ}$ ). A partir dos resultados obtidos espera-se que se possa consolidar uma metodologia para avaliação do melhor ângulo possível para a linha de solda de um TWB. Constatou-se que a carga máxima suportada aumenta à medida que se inclina a linha de solda (até um determinado limite) e que ângulos complementares de inclinação geram cargas cisalhantes similares. Deve-se trabalhar então com ângulos que permitam otimização de resistência mecânica, melhor conformabilidade e maior redução de massa e de custos. A seleção desse ângulo ficará atrelada a questões de projeto e não mais a tentativas e erros.
\end{abstract}

Palavras-chave: Conformação; Soldagem a laser; Tailor Welded Blank; Indústria automobilística.

\section{Mechanical Characterization and Micro-structural Analysis of Plates Obtained by the Tailor Welded Blank process (TWB)}

\begin{abstract}
One of the biggest challenges facing the auto industry globally is to develop vehicles that are both lightweight and mechanically resistant. Tailor Welded Blank (TWB) comes as a solution as it is a process that allows butt welding of sheets of different specifications (material, thickness and / or coating) prior to forming. In this research we worked with Interstitial Free (IF) steel plates of the Companhia Siderúrgica Nacional (CSN), with FEE 210 codes of 1.2 mm thickness and IF CSN FEP 05 of $0.7 \mathrm{~mm}$ thickness, welded. Laser Uniaxial tensile and Vickers microhardness tests were performed. The weld line was oriented from $0^{\circ}$ to $70^{\circ}$ (ranging from $5^{\circ}$ to $5^{\circ}$ ). From the results obtained it is expected that a methodology can be consolidated to evaluate the best possible angle for the weld line of a TWB. It has been found that the maximum load supported increases as the weld line is tilted (up to a certain limit) and that additional bending angles generate similar shear loads. Then work with angles that allow optimization of mechanical strength, better conformability and greater mass and cost reduction. Selecting this angle will be tied to design issues.
\end{abstract}

Key-words: Forming; Laser welding; Tailor Welded Blank; Auto industry.

\section{Introdução}

Umas das indústrias mais importantes para o desenvolvimento tecnológico global é a automobilística. Nesta indústria trabalha-se com pesquisas em novos materiais, tecnologias e processos, buscando a cada dia, atender demandas mais restritivas de emissão de poluentes e de consumo energético e de combustível, exigindo de forma crescente redução de massa e aumento de desempenho estrutural dos os automóveis.

Quanto aos processos mecânicos, no ramo automobilístico o mais empregado é a conformação por estampagem, sendo um dos mais antigos processos de fabricação de materiais, nas suas mais diversas técnicas e formas [1-4]. 
A maioria das peças que compõem a carroceria de um automóvel são obtidas por meio de estampagem e unidas por soldagem. Via de regra, para se reduzir a massa, acaba-se perdendo em desempenho estrutural. Uma alternativa para que se minimize o ganho de peso é, apenas em regiões de maiores solicitações mecânicas, utilizar reforços ou batentes $[5,6]$.

Ainda que se reforce apenas as regiões de maior solicitação mecânica, o acréscimo de massa ainda é prejudicial à eficiência energética do veículo. Uma das maneiras de conciliar então esses conceitos antagônicos (redução de massa $\times$ desempenho estrutural) é utilizar o processo de fabricação por Tailor Welded Blanks (TWBs), conforme apontam os estudos mais recentes [7-13].

A técnica de TWBs consiste basicamente em fabricar blanks semiacabados, compostos por ao menos duas chapas distintas (em termos de material, espessura, revestimentos e outras propriedades física e/ou químicas), antes da etapa de conformação final para obtenção do produto acabado [14-16]. Por meio do processo de TWB consegue-se confeccionar peças com espessura diferenciada na região de maior solicitação mecânica, dispensando o uso de reforços e componentes adicionais.

O estudo de TWBs, focado nas variáveis do processo de soldagem e nas operações posteriores de conformação é crescente, visando otimização e difusão da tecnologia. Ainda assim se trabalha, em diversas aplicações, com métodos empíricos. Tem-se como relevância e justificativa do estudo o fato de que inicialmente o processo de Tailor Welded Blank apresentava elevados custos, o que inviabilizava sua aplicação em larga escala, como na indústria automobilística [17-19]. Essa tecnologia avançou de forma gradual e tem sido empregada não apenas em veículos, como em outros setores estratégicos: construção e indústria aeroespacial, por exemplo [20].

Não se trabalha, na maioria dos casos, com a orientação da solda e sua relação de otimização entre comportamento mecânico, redução de massa e de custos do blank [21]. O presente estudo firma-se, portanto, na necessidade de conferir mais técnica e ciência ao processo de fabricação por TWBs, que muitas vezes é feito de forma empírica [4,6,21]. Pretende-se então avaliar o comportamento de diferentes chapas obtidas pelo processo de Tailor Welded Blank (soldadas a laser) ante ao processo de conformação. A resistência à tração, o perfil de microdureza Vickers (HMV) e a orientação da solda em relação à aplicação da carga serão levadas em consideração.

\section{Metodologia}

Foram executados ensaios de tração uniaxial e de microdureza Vickers. Trabalhou-se com amostragem em triplicata, para maior confiabilidade dos resultados. As análises microestruturais foram feitas com auxílio de microscópio óptico. As chapas utilizadas foram cedidas, já soldadas, pela FIAT Chrysler Automóveis (FCA) Brasil. Os parâmetros de soldagem utilizados foram:

- Equipamento: $\mathrm{CO}_{2}$ SoundRonic ${ }^{\circledR}$ com potência nominal de $8 \mathrm{~kW}$;

- Potência média utilizada: $65 \%$;

- Gás de proteção: He puro $(10,5 \mathrm{~L} / \mathrm{min})$;

- Velocidade de soldagem: $6,5 \mathrm{~m} / \mathrm{s}$.

Uma das chapas cedidas foi estampada, também pela FCA Brasil, para fins de análise dos efeitos da solda, em diferentes regiões, sobre a conformabilidade dos materiais. Na Figura 1 apresenta-se um fluxograma das etapas executadas nesta pesquisa.

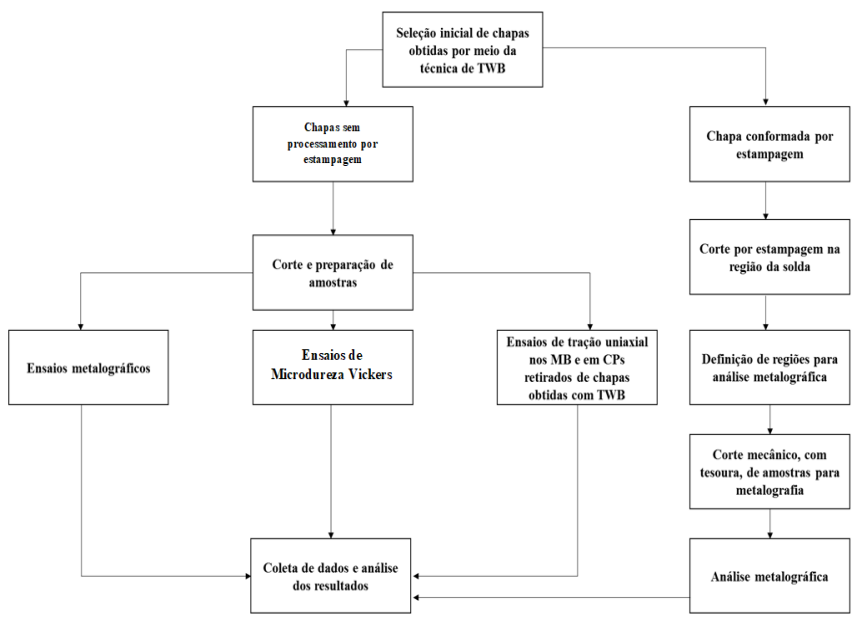

Figura 1. Fluxograma de etapas desenvolvidas na presente pesquisa. $\mathrm{MB}=$ Metal e base $; \mathrm{CP}=$ Corpo de prova.

Para compor o TWB foram utilizados os materiais apresentados nas Tabelas 1 e 2, cujos dados informados foram fornecidos pelo fabricante: 
Tabela 1. Composição química dos aços IF utilizados no TWB [22].

\begin{tabular}{cccccccccc}
\hline Elemento (\%) & $\mathbf{C}$ & $\mathbf{M n}$ & $\mathbf{P}$ & $\mathbf{S}$ & $\mathbf{S i}$ & $\mathbf{N}$ & $\mathbf{N b}$ & $\mathbf{T i}$ \\
IF FEP 05 & 0,0018 & 0,1350 & 0,0120 & 0,0100 & 0,0050 & 0,00420 & 0,0030 & 0,0570 \\
IF FEE 210 & 0,0021 & 0,4320 & 0,0360 & 0,0120 & 0,0050 & 0,00360 & 0,0024 & 0,0260 \\
\hline
\end{tabular}

Tabela 2. Propriedades mecânicas dos aços IF utilizados no TWB [22].

\begin{tabular}{cccc}
\hline Material e espessura da chapa & LE [MPa] & LRT [MPa] & Expoente de encruamento (n) \\
IF CSN FEE $210-1,2 \mathrm{~mm}$ & 217 & 346 & 0,21 \\
IF CSN FEP05 $-0,7 \mathrm{~mm}$ & 157 & 284 & 0,25 \\
\hline
\end{tabular}

$\mathrm{LE}$ = Limite de escoamento; $\mathrm{LRT}$ = Limite de Resistência à Tração; $\mathrm{n}$ = Expoente de encruamento.

Embora, conforme apontado pelos estudos de Abbasi et al. [23], Ahmetoglu et al. [24] e Choi et al. [25], não se tenha uma regra específica para determinação da espessura das chapas que comporão o TWB, trabalha-se com base no projeto que motivou o emprego dessa técnica. Na região destinada à ancoragem da dobradiça da porta dos veículos soldava-se um batente/reforço. Por esse motivo, optou-se por trabalhar, nesse local, com uma chapa mais espessa e de maior resistência mecânica.

Uma das chapas planas foi selecionada e submetida ao processo de conformação por estampagem. Foram executadas cinco etapas de estampagem, a saber: repuxo (confere a forma inicial do produto); corte primário (recorta o perímetro do vão de vidro, com auxílio de uma cunha); corte secundário e furação (recorta detalhes e executa furações); calibração (calibra e ajusta dimensões e detalhes do produto) e furação final (recorta últimos retalhos, confere detalhes e furações finais).

Após essas operações, a fim de avaliar a solda e seu entorno, o painel interno de porta foi recortado a $80 \mathrm{~mm}$ à direita e à esquerda do centro da solda. A chapa foi inicialmente dividida em três regiões distintas, conforme apresentado na Figura 2.

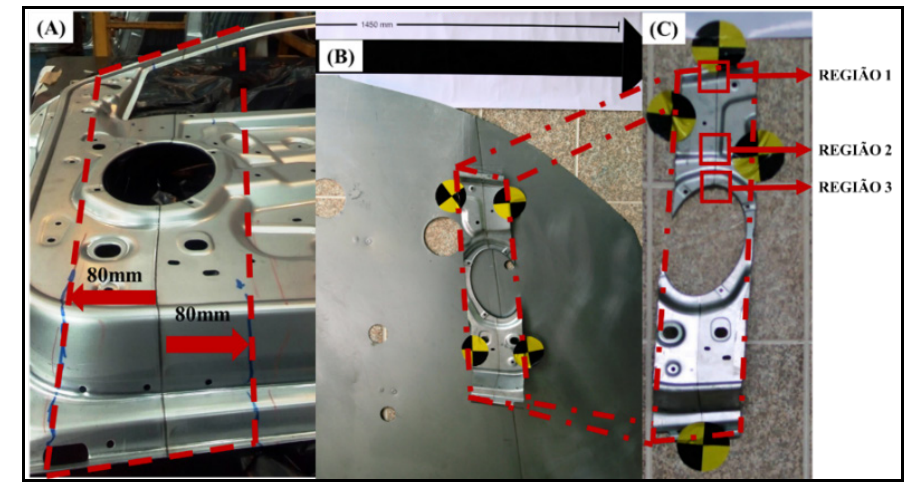

Figura 2. (A) Painel interno de porta, com detalhe para região de corte (B) peça cortada, sobreposta à chapa plana de TWB e (C) peça após estampagem e corte, com destaque para regiões analisadas.

Para caracterização mecânica do TWB foram feitos ensaios de tração, também conforme determina norma ASTM E-8M [26], optando-se pelo tamanho padrão conforme apresentado na Figura 3. Os testes foram executados em conjunto ao Instituto SENAI de Tecnologia Metal Mecânica (ISTMM). Utilizou-se uma máquina de ensaios da marca KRATOS ${ }^{\circledR}$, modelo KE, com célula de carga de $5 \mathrm{kN}$. Trabalhou-se com extensômetro óptico e taxa de deformação de $0,001 \mathrm{~s}^{-1}$.

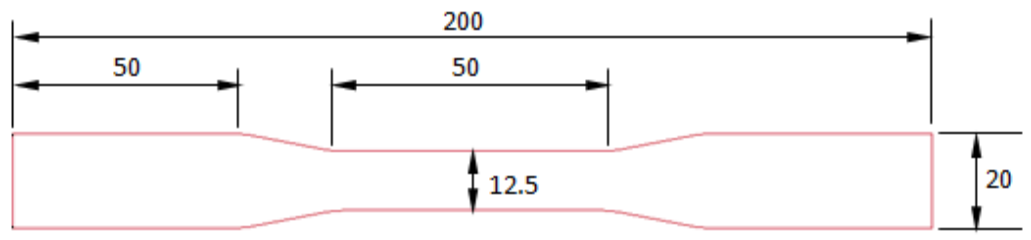

Figura 3. Dimensões do corpo de prova padrão para ensaios de tração segundo norma ASTM E- 8M [26]

Conforme mencionado, muitos dos testes realizados ou são meramente qualitativos ou focam apenas na conformabilidade, em si. O presente trabalho focou na avaliação da influência da orientação da linha de solda nas propriedades mecânicas do TWB. Ghoo et al. [27], Xu et al. [28] e Kim et al. [29] defendem que os ensaios de tração podem ser significativos. 
Caso se opte por orientar a linha de solda em ângulos diferentes dos padrões ( $0^{\circ} \mathrm{DL}, 45^{\circ} \mathrm{DL}$ e $\left.90^{\circ} \mathrm{DL}\right)$, os resultados se tornam mais complexos e representativos.

Optou-se então por retirar as amostras diretamente da chapa que compõe o TWB utilizado na fabricação de painéis de portas dianteiras. Para isso trabalhou-se com o processo de corte a jato de água, a fim de evitar a geração de calor nas proximidades da solda, o que interferiria nos resultados. Os corpos de prova foram orientados, em relação à posição inicial da solda, com angulação variando de $0^{\circ}$ a $70^{\circ}$. Os valores dos ângulos foram aumentados a cada $5^{\circ}$. Todos os ensaios foram realizados em triplicata. Não se optou por um ângulo superior a $70^{\circ}$ pois a partir de $75^{\circ}$ as cabeças dos corpos de prova passaram a ocupar ambas as chapas do TWB, possuindo espessuras distintas. Isso dificultaria a fixação adequada nas garras de tração, comprometendo o ensaio. Quanto à disposição dos resultados, boa parte dos artigos pesquisados não deixa claro como são calculados os limites de escoamento e de resistência mecânica. Como se tem três espessuras distintas no mesmo corpo de prova (chapa de menor espessura, ZF - Zona Fundida e chapa mais espessa), não fica claro qual a área da seção transversal utilizar.

Embora a ruptura seja esperada na chapa menos espessa, isso nem sempre acontece. Em virtude dessas dificuldades optou-se por tratar os resultados apenas em função da carga de ruptura suportada por cada corpo de prova. Na Figura 4 são apresentados alguns CPs utilizados, com destaque para região de angulação da solda. Neste contexto considerou-se a decomposição da carga de ruptura em carga puramente de tração e em carga cisalhante foi baseada no princípio de variação da tensão/carga com o ângulo do plano de corte, mostrado por Callister e Rethwisch [30], Dieter [31] e Hosford e Caddell [32]. Foram utilizadas as Equações 1 e 2.

$\sigma^{\prime}=\sigma \cos ^{2} \theta$

$\tau^{\prime}=\sigma \sin \theta \cos \theta$

Nas quais:

- $\sigma=$ Tensão puramente de tração [MPa];

- $\sigma^{\prime}=$ Tensão normal [MPa];

- $\tau^{\prime}=$ Tensão cisalhante [MPa];

- $\Theta$ = Ângulo relativo de inclinação do plano de corte [ ${ }^{\circ}$ ou rad].

Além disto considerou-se que o aumento da carga, ao se aumentar o ângulo da solda, pode ser explicado pela maior área ocupada pela solda, de acordo com a Equação 3.

$A_{s}=\frac{L_{C P}}{\cos \delta} \cdot e_{S}$

Na qual:

- $\mathrm{A}_{\mathrm{s}}=$ Área ocupada pela solda $\left[\mathrm{mm}^{2}\right]$;

- LCP = Largura útil do corpo de prova de tração [mm];

- $\delta$ = Ângulo de inclinação relativa da solda $\left[^{\circ}\right]$;

- $\mathrm{e}_{\mathrm{s}}=$ Espessura média da solda [mm].

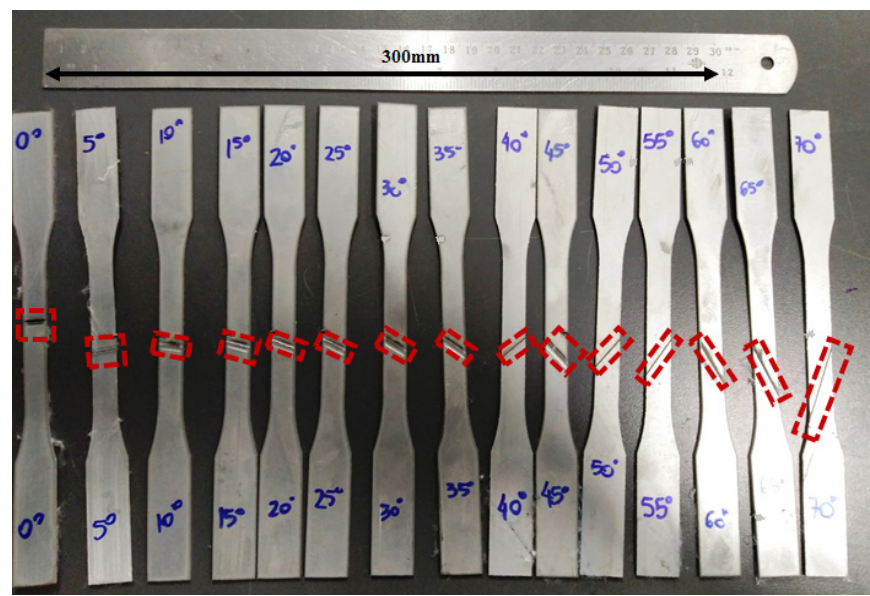

Figura 4. Corpos de prova utilizados para execução dos ensaios de tração, com destaque para região da solda. 
Os ensaios de microdureza Vickers foram realizados conforme previsto nas normas ASTM E-92 [33] e ASTM E-384 [34]. Com base nos trabalhos de Panda et al. [35] e de Li et al. [3] e em testes preliminares determinou-se carga de endentação de 300g.f, por um período de 15s. Na Figura 5 está representado, de forma esquemática, o método utilizado para coleta de dados e traçagem do perfil de microdureza Vickers. Os ensaios também foram realizados em triplicata. Inferiu-se a extensão das ZTAs (Zonas Termicamente Afetadas) e da ZF por meio do perfil de microdureza e avaliou-se a homogeneidade dos materiais de base.

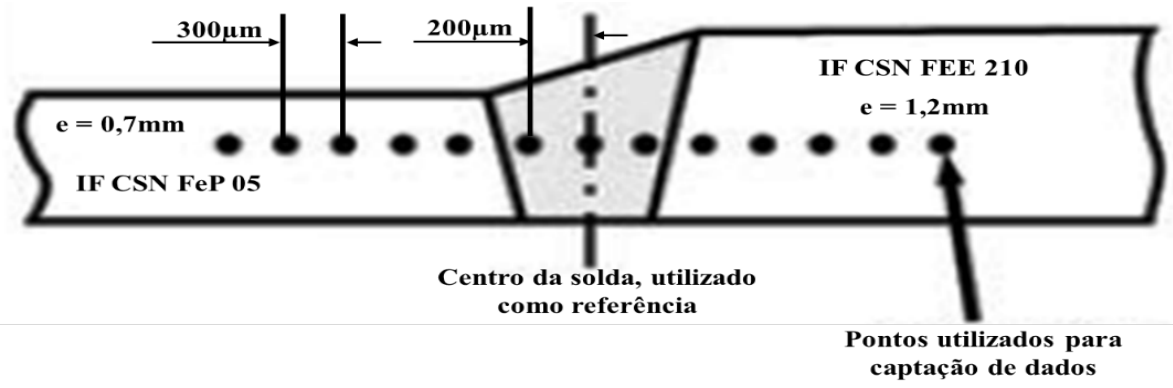

Figura 5. Representação esquemática do método utilizado para coleta de dados de microdureza Vickers. e = Espessura das chapas que compõem o Tailor Welded Blank utilizado no estudo [36].

As análises metalográficas foram feitas seguindo recomendações da norma ASTM E-3 [37], Colpaert [38] e de artigos científicos utilizados para elaboração das referências. Foram retiradas amostras diretamente do blank, de perfil e de topo. A fim de avaliar o efeito da angulação relativa da solda na microestrutura e da área da linha de solda, foram confeccionadas amostras com diferentes ângulos de inclinação em relação à posição inicial da solda. Essas amostras foram analisadas de perfil, com ângulos relativos de $15^{\circ}, 20^{\circ}, 25^{\circ}, 30^{\circ}, 40^{\circ}, 45^{\circ}$ e $60^{\circ}$.

Após a retirada das amostras, optou-se pelo método de embutimento a frio, com resina epóxi. Trabalhou-se com a resina Cristal 5061 e com o catalisador MEK. A resina foi preparada na proporção de 30 gotas de catalisador para cada $100 \mathrm{~g}$ de resina. Para pesagem das quantidades utilizou-se uma balança de precisão BEL Engineering ${ }^{\circledR} \mathrm{M} 214$.Para preparação das amostras após o embutimento foram utilizadas lixadeiras automáticas circulares e manuais de bancada. O lixamento com lixas \#80, \#150 e \#320 ocorreu nas lixadeiras automáticas e com as lixas \#600, \#1200, \#1500 e \#2000, na de bancada. Em seguida prosseguiu-se, com politriz circular automática, o polimento com alumina em suspensão de $1 \mu \mathrm{m}$. Após o polimento as amostras foram lavadas com álcool isopropílico e água e secadas com secador. $\mathrm{O}$ ataque químico foi realizado com Nital a $5 \%$, utilizando a técnica de esfregaço, com cerca de 10 esfregaços em cada sentido. Logo após o ataque as amostras foram limpas também com álcool isopropílico e água e posteriormente secas com secador.

As análises microestruturais foram realizadas com um microscópio óptico modelo Olympus ${ }^{\circledR}$ BX $60 \mathrm{MF5}$. Sobre o microscópio foram acopladas uma lente objetiva de 10X de aumento Olympus ${ }^{\circledR}$ U-TV 0.5XC e uma câmera digital de alta definição TOUPCAM ${ }^{\mathrm{TM}}$ UCMOS 03100KPA. A coleta de dados ocorreu através de um cabo USB diretamente acoplado à lateral da câmera de alta definição. Utilizou-se o software ToupTek ${ }^{\circledR}$ ToupView x64 para tratamento inicial das imagens. Para cada amostra selecionada, a fim de percorrer os metais de base, a ZTA e a ZF foram extraídas cerca de 30 imagens. Para correção, tratamento e união das imagens trabalhou-se com o software Adobe ${ }^{\circledR}$ PhotoShop CS5. O software Image $^{\circledR}$ foi utilizado para análises dimensionais, inserção e calibração de barras de escala.

\section{Resultados e Discussão}

Na Figura 6 estão apresentadas as relações entre a carga de ruptura (A), a carga puramente de tração (B), a carga cisalhante (C), a deformação verdadeira (D) e o ângulo de inclinação relativa da solda, para os diferentes corpos de prova de tração de TWB testados. 

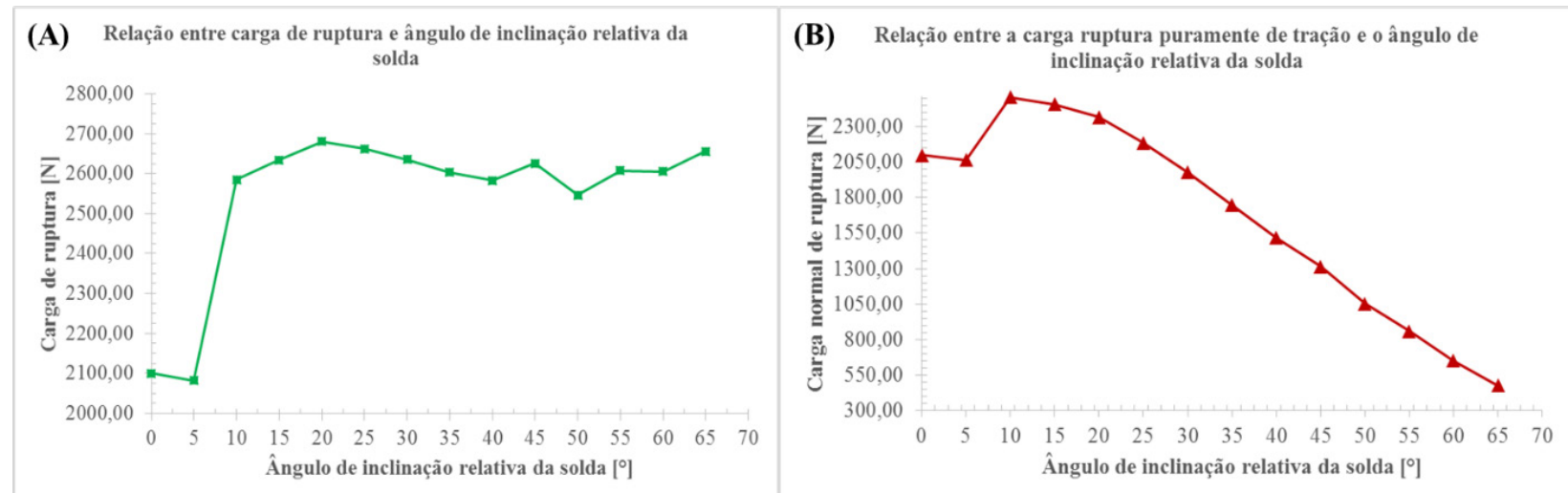
(C) Relação entre a carga cisalhante e 0 ângulo de inclinação relativa

(D) Deformação verdadeira em função do ângulo de inclinação relativa
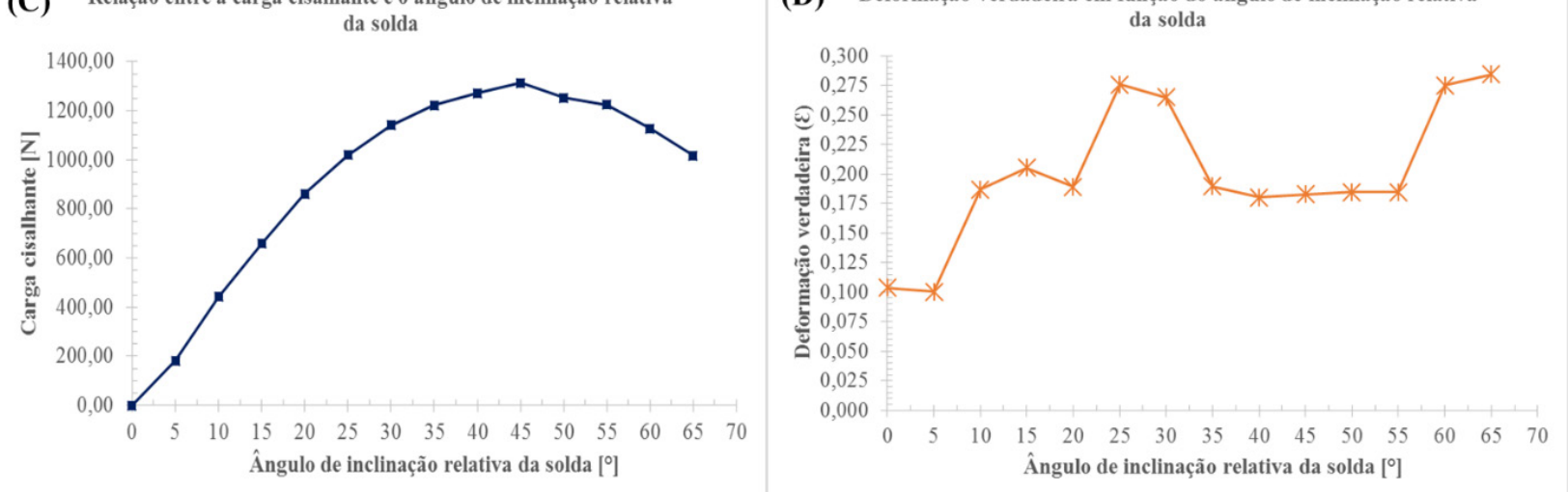

Figura 6. Relações entre a carga de ruptura (A), a carga puramente de tração (B), a carga cisalhante (C), a deformação verdadeira (D) e o ângulo de inclinação relativa da solda, para os diferentes corpos de prova de tração de TWB testados. $\varepsilon=$ Deformação verdadeira; $\mathrm{N}=$ Newtons.

Verifica-se que um aumento abrupto de carga limite de ruptura quando o ângulo de inclinação relativa da solda ultrapassa os $10^{\circ}$. Para o comportamento exibido entre $0^{\circ}$ e $5^{\circ}$, discrepante em relação aos demais ângulos, descarta-se, pela triplicata, a hipótese de erros experimentais. Esse comportamento não era esperado e pode estar associado a questões do material em si ou de alguma condição associada especificamente a esses ângulos da solda. O valor máximo de carga é atingido por volta dos $20^{\circ}$ de inclinação, a partir do instante em que há leve queda. Entre $45^{\circ}$ e $50^{\circ}$ tem-se uma relativa piora. Os valores de carga para os ângulos subsequentes são similares aos seus ângulos complementares. Para $70^{\circ}$, por exemplo, tem-se uma carga muito similar à de $20^{\circ}$.

Sendo assim, quanto maior o ângulo de inclinação relativa, maior tende a ser a área ocupada pela solda. Como se espera que a solda apresente maior limite de resistência à tração que ambos os materiais de base, a carga de ruptura tende então a aumentar. No entanto, esse comportamento não é linear. Conforme apontado por Gong et al. [39], Kim et al. [18] e Zhang et al. [40], a solda nem sempre é homogênea podendo conter micro trincas ou poros, tampouco o material que pode conter inclusões, diferentes tamanhos de grãos etc. A inclinação relativa pode induzir no corpo de prova outras tensões que não a puramente de tração, o que também contribui com a não linearização do comportamento. Lida-se com anisotropia e extensão e microestrutura relativa da Zona Termicamente Afetada (ZTA).

Não há, no entanto, consenso quanto aos fatores que podem afetar comportamento mecânico de Tailor Welded Blanks com o posicionamento relativo da linha de solda. Isso é apontado nos trabalhos de Gong et al. [39], Li et al. [6], Assunção et al. [41] e reafirmado por Riahi et al. [21]. Verifica-se, conforme previsto por Cetlin e Helman [42], Callister e Rethwisch [30] e Hosford e Caddell [32], que há uma tendência de que, à medida que se inclina a solda, a tensão puramente de tração diminua.

Isso porque, conforme a Equação 1, um aumento no ângulo gera uma diminuição em seu cosseno, chegando-se a um limiar de nulidade para tração puramente dita, ao se trabalhar com um plano de corte a $90^{\circ}$. Esses resultados estão condizentes com os trabalhos realizados por Li e Lin [43], Riahi et al. [21] e Zadpoor et al. [17]. Porém, esperava-se que a pior condição ocorresse com um ângulo de $45^{\circ}$, conforme apontado Narayanan e Naik [44]. Isso, no entanto, não ocorreu porque os referidos trabalhos executaram apenas testes de embutimento, nos quais o material sujeita-se ao estiramento e à uma condição multiaxial de tensões. Esse estado de tensões e deformações tende a ser agravado com a solda orientada a $45^{\circ}$.

De acordo com Parente et al. [13], um bom ângulo de orientação seria o de $90^{\circ}$. No entanto, os resultados mostram que, caso continue-se com a tendência apresentada na Figura 6A e 6B, isso não seria verdade. Essa afirmativa contraria também as boas práticas de soldagem e os trabalhos apresentados por Riahi et al. [21], Kinsey et al. [45] e Li et al. [3], os quais orientam 
que a solda não deve ser posicionada paralelamente à direção de laminação. Caso isso ocorra, trincas podem se propagar com mais facilidade, percorrendo todo o entorno da linha de solda, sem encontrar obstáculos.

Verifica-se que a carga cisalhante, conforme indicado pela Equação 2, assume seu valor de máximo para um ângulo de $45^{\circ}$. Apesar de aparentemente simples, essa constatação não é trivial. Matematicamente, ângulos complementares possuem valores inversos de cosseno e de seno $\left(\operatorname{sen} 30^{\circ}=\cos 60^{\circ}\right)$. Daí, de acordo $\operatorname{com}$ a Equação 2 , o produto sen $\Theta \cdot \cos \theta$ é idêntico para ângulos complementares. Ocorre, no entanto, que há também a dependência da carga aplicada sobre o plano de corte, o que aparentemente independe do ângulo de inclinação relativa da solda. Porém, de acordo com os resultados apresentados na Figura $6 \mathrm{~A}$, esses valores de carga seguem um comportamento similar ao produto sen $\Theta . \cos \Theta$. Em decorrência disso a tensão cisalhante exibe o padrão apresentado na Figura 6C.

Esse padrão não foi encontrado em nenhum dos artigos pesquisados. Talvez porque a maioria dos trabalhos não ultrapasse as análises com $0^{\circ} \mathrm{DL}, 45^{\circ} \mathrm{DL}$ e $90^{\circ} \mathrm{DL}$. No estudo desenvolvido por Li et al. [3] foram feitos corpos de prova com ângulos diferentes $\left(30^{\circ}\right.$ e $\left.60^{\circ}\right)$. No entanto, não se focou na análise da tensão cisalhante, apenas na deformação, para alimentação da técnica de correlação digital de imagem.

Outras pesquisas similares, como as desenvolvidas por Riahi et al. [21] e por Safdarian [46], deslocaram a linha de solda em relação ao centro do TWB. Constatou-se que, em termos de tração, praticamente não há diferença perceptível entre os resultados. No entanto, em ambos os trabalhos se observou relativa piora de conformabilidade ao se trabalhar com o teste de altura limite do domo (LDH), quando se desloca a linha de solda em direção ao material de menor resistência mecânica. Para análise do padrão apresentado na curva de tensão cisalhante da Figura $6 C$ verificou-se a tendência da deformação verdadeira exibida pelos diferentes corpos de prova.

De acordo com os resultados dispostos na Figura 6D, pode-se verificar que os menores valores de deformação são obtidos quando a solda praticamente não exibe inclinação relativa $\left(0^{\circ}\right.$ e $\left.5^{\circ}\right)$. Esse comportamento é similar ao da carga limite de ruptura. Isso porque, como se trabalha com materiais relativamente dúcteis, espera-se que quanto maior a carga de ruptura, maior tenderá a ser a deformação. Nota-se mais uma vez a similaridade de valores de deformação para ângulos complementares. Conforme apontado por Dowling [47], Dharan et al. [48] e Affonso [49], na teoria da Mecânica da Fratura, quando um material apresenta matriz relativamente dúctil, sua fratura é determinada primordialmente pelas tensões de cisalhamento que atuam em seus planos de deslizamento. Ressalta-se que observações similares foram constatadas por Li e Lin [43], trabalhando com a solda orientada a $30^{\circ}, 45^{\circ}, 60^{\circ}$ e $90^{\circ}$. O foco do trabalho foi o emprego da técnica de correlação digital de imagens.

Na Figura 7 apresenta-se o perfil médio de microdureza Vickers das chapas obtidas pelo processo de TWB.

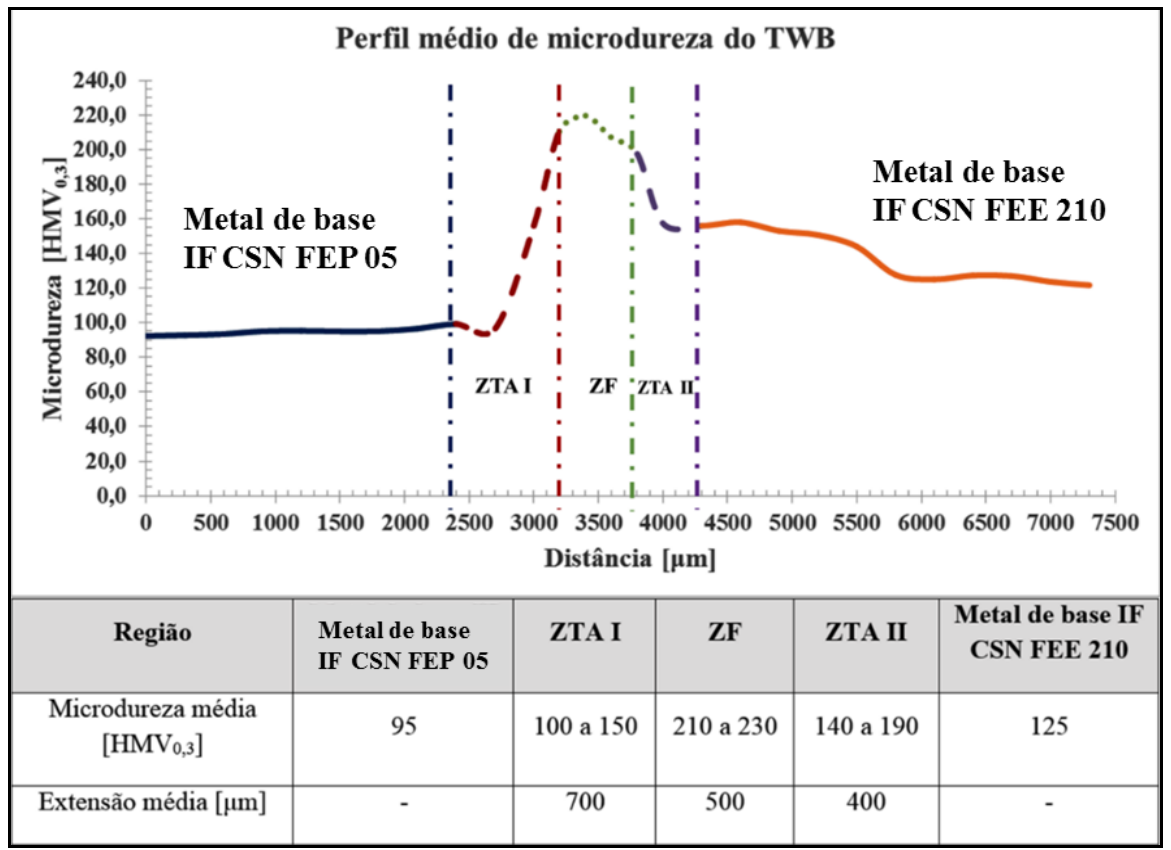

Figura 7. Perfil médio de microdureza Vickers, com delimitação de regiões da solda do TWB segundo perfil médio de microdureza Vickers. ZTA = Zona Termicamente Afetada; ZF = Zona Fundida.

O comportamento apresentado no gráfico da Figura 7 é típico de um TWB composto de aços IF, soldado a laser. Perfis similares foram encontrados nos trabalhos de Safdarian Korouyeh et al. [50] e Min et al. [51]. A relativa distorção presente no gráfico a partir de $5500 \mu \mathrm{m}$ deve-se provavelmente a três fatores: erros de medição, heterogeneidades do material e método de corte manual utilizado para preparação da amostra. Embora os artigos apresentados não façam essa estimativa, optou-se por fazê-la a fim de analisar o efeito da soldagem em ambos os materiais de base. Pode-se verificar que o material de base IF CSN 
FEE 210 é, de fato, mais resistente mecanicamente. Talvez por esse fator o crescimento de grão seja mais limitado e sua ZTA tenha menor extensão que a do IF CSN FEP05. A ZF apresenta extensão média de $500 \mu \mathrm{m}$ e sua maior dureza deve-se, conforme apontado pelos trabalhos de Min et al. [51] e Safdarian Korouyeh et al. [50] e por Krauss [52] e Callister e Rethwisch [30] à formação de bainita e de precipitados duros de Ti, $\mathrm{Nb}$ e TiNb.

Na Figura 8 apresenta-se uma micrografia das chapas fabricadas a partir do processo de TWB.
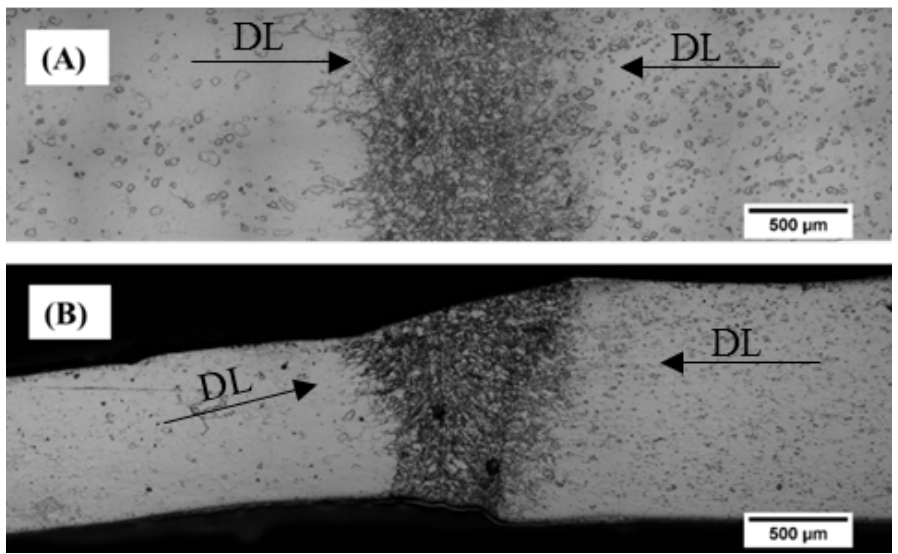

Figura 8. Micrografia de topo (A) e de perfil (B) das chapas obtidas pelo processo de TWB. DL = Direção de Laminação.

A microestrutura exibe, em ambos os materiais de base, matriz ferrítica. Verifica-se também que a soldagem, como apresentado na Metodologia, foi feita perpendicularmente à direção de laminação de ambos os materiais. $O$ material da chapa menos espessa, IF CSN FEP05, apresenta grãos ligeiramente maiores e marcações de microdureza Vickers menores. Isso é compatível com o apresentado por Colpaert [38] e Callister e Rethwisch [30] e com o observado nos trabalhos de Min et al. [51] e Safdarian Korouyeh et al. [50]. Quanto maiores os teores de carbono e de manganês, maior tende a ser a dureza do aço IF. O IF CSN FEE 210 também apresenta maior quantidade de fósforo e menores teores de titânio e de nióbio. Esse conjunto de fatores pode ser o responsável pelo menor tamanho de grão, maior dureza e propriedades mecânicas relativamente superiores desse material em relação ao IF CSN FEP 05.

Na Figura 9 apresentam-se micrografias de topo das amostras conformadas de diferentes regiões do painel de porta interno, próximo à solda do TWB.

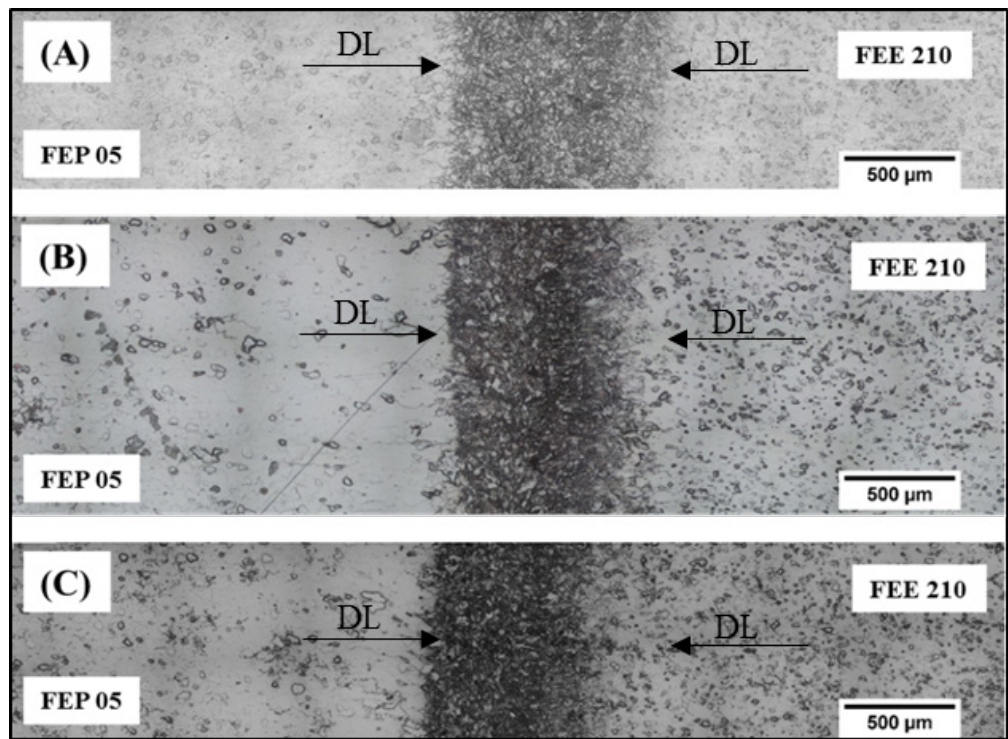

Figura 9. Micrografias de topo de amostras conformadas. (A) Região 1, (B) Região 2 e (C) Região 3. DL = Direção de Laminação.

De acordo com Dieter [31] e Hosford e Caddell [32], quanto maior o expoente de encruamento, maior tende a ser o encruamento do material. Apesar da ligeira diferente entre os expoentes de encruamento do aço IF CSN FEP 05 ( $n=0,25$ ) e do IF CSN FEE $210(n=0,21)$, pode-se notar essa diferença durante a conformação. Isso porque a chapa menos espessa, feita de IF CSN FEP 05 flui muito mais do que a chapa mais espessa. Esse fenômeno ocorre porque, durante a conformação, ambas as 
chapas se sujeitam à mesma pressão de estampagem (600ton.f no prensa chapas e 400ton.f no punção). Como o FEP 05 possui menor resistência mecânica que o FEE 210, acaba se deformando mais.

Nota-se também que os grãos se deformam mais à medida que há maior solicitação mecânica durante a conformação. Isso pode ser percebido por meio da diferença entre o tamanho médio dos grãos à medida que se muda da região 1 (submetida praticamente apenas à sujeição do prensa-chapas) para região 3 (submetida a um maior número de operações de estampagem e à maior deformação plástica). Pode-se esperar que num TWB a região de menor espessura seja não apenas aquela que falhará preferencialmente, mas também aquele que deve ser submetida às operações de conformação mais complexas.

\section{Conclusões}

Por meio de ensaios de tração com diferentes ângulos relativos da solda, análise de perfis de microdureza Vickers e ensaios metalográficos, pôde-se concluir que:

- À medida que se aumenta a angulação da solda tende-se a aumentar a carga de ruptura dos corpos de prova de tração;

- O aumento da carga de ruptura dos corpos de prova de tração deve-se principalmente à diminuição da carga normal de tração, a qual depende principalmente do cosseno do ângulo de inclinação do corpo de prova de tração;

- Corpos de prova com ângulos complementares apresentam perfil geométrico da fratura e deformação semelhantes. Isso indica que, para fins de aumento da redução de massa e otimização da utilização de material pode-se optar por ângulos complementares, sendo as cargas equivalentes;

- $\quad$ Ao se angular a solda, os grãos da ZTA também seguem tal perfil de angulação. Isso pode explicar o aumento da carga cisalhante e da carga de ruptura: aumenta-se a extensão da solda, a qual tende a retardar o início da instabilidade plástica do TWB;

- De acordo com as condições analisadas, tendo em base os ensaios executados, as chapas fornecidas e os resultados obtidos, o ângulo mais adequado de trabalho (que permite boa relação entre carga cisalhante e de ruptura) para se soldar o TWB é em torno de $25^{\circ}$.

Por meio da revisão bibliográfica, percebeu-se que o posicionamento relativo da linha de solda em chapas obtidas por meio de TWBs é feito de forma aleatória. Busca-se primordialmente que a chapa não falhe e que se tenha a menor massa possível, aliada à redução de custos de fabricação.

Como se constatou que a carga necessária à ruptura das chapas aumenta à medida que se inclina a linha de solda (até um determinado limite) e que ângulos complementares de inclinação geram cargas cisalhantes similares, espera-se que isso seja levado em consideração em projetos futuros.

Deve-se trabalhar com ângulos que permitam otimização de resistência mecânica, melhor conformabilidade e maior redução de massa e de custos. A seleção do melhor ângulo de inclinação relativa da linha de solda ficará atrelada a questões de projeto e não mais apenas a tentativas e erros.

\section{Agradecimentos}

Os autores agradecem à FIAT Chrysler do Brasil pela contribuição técnica e seção de materiais para presente pesquisa.

\section{Referências}

[1] Suresh VVNS, Regalla SP, Gupta AK. Combined effect of thickness ratio and selective heating on weld line movement in stamped tailorwelded blanks. Materials and Manufacturing Processes. 2017;32(12):1363-1367. http://dx.doi.org/10.1080/10426914.2016.1257128.

[2] Duan L, Xiao N-C, Li G, Xu F, Chen T, Cheng A. Bending analysis and design optimization of tailor-rolled blank thin-walled structures with top-hat sections. International Journal of Crashworthiness. 2017;22(3):227-242. http://dx.doi.org/10.1080/13588265.2016.1246069.

[3] Li G, Xu F, Huang X, Sun G. Topology optimization of an automotive tailor-welded blank door. Journal of Mechanical Design. 2015;137(5):055001-055008. http://dx.doi.org/10.1115/1.4028704.

[4] Mohebbi MS, Akbarzadeh A. Prediction of formability of tailor welded blanks by modification of MK model. International Journal of Mechanical Sciences. 2012;61(1):44-51. http://dx.doi.org/10.1016/j.ijmecsci.2012.05.001.

[5] Gaied S, Roelandt J-M, Pinard F, Schmit F, Balabane M. Experimental and numerical assessment of Tailor-Welded Blanks formability. Journal of Materials Processing Technology. 2009;209(1):387-395. http://dx.doi.org/10.1016/j.jmatprotec.2008.02.031.

[6] Li J, Nayak SS, Biro E, Panda SK, Goodwin F, Zhou Y. Effects of weld line position and geometry on the formability of laser welded high strength low alloy and dual-phase steel blanks. Materials \& Design. 2013;52:757-766. http://dx.doi.org/10.1016/j.matdes.2013.06.021.

[7] Chan LC, Chan SM, Cheng CH, Lee TC. Formability and weld zone analysis of tailor-welded blanks for various thickness ratios. Journal of Engineering Materials and Technology. 2005;127(2):179-185. http://dx.doi.org/10.1115/1.1857936. 
[8] Gautam V, Raut VM, Kumar DR. Analytical prediction of springback in bending of Tailor-Welded Blanks incorporating effect of anisotropy and weld zone properties. Proceedings of the Institution of Mechanical Engineers, Part L: Journal of Materials: Design and Applications. 2018;232(4):294-306. http://dx.doi.org/10.1177/1464420715624261.

[9] Liu J, Wang L-L, Lee J, Chen R, El-Fakir O, Chen L, et al. Size-dependent mechanical properties in AA6082 tailor welded specimens. Journal of Materials Processing Technology. 2015;224:169-180. http://dx.doi.org/10.1016/j.jmatprotec.2015.05.004.

[10] Gan Y, Li JX, Chen Y, Li HZ. The optimization method of tailor welded blank forming process based on numerical simulation. Applied Mechanics and Materials. 2014;721:135-139. http://dx.doi.org/10.4028/www.scientific.net/AMM.721.135.

[11] Kumar TK, Reddy KJ. Tailor Welded Blanks. International Journal of Research in Engineering and Technology. 2015;4(2):63-72.

[12] Wang H, Zhou J, Zhao TS, Liu LZ, Liang Q. Multiple-iteration springback compensation of tailor welded blanks during stamping forming process. Materials \& Design. 2016;102:247-254. http://dx.doi.org/10.1016/j.matdes.2016.04.032.

[13] Parente M, Safdarian R, Santos AD, Loureiro A, Vilaca P, Jorge RMN. A study on the formability of aluminum tailor welded blanks produced by friction stir welding. International Journal of Advanced Manufacturing Technology. 2016;83(9-12):2129-2141. http://dx.doi.org/10.1007/s00170-015-7950-0.

[14] Zhang Y-X, Han S-W, Cheon J, Na S-J, Gao X-D. Effect of joint gap on bead formation in laser butt welding of stainless steel. Journal of Materials Processing Technology. 2017;249:274-284. http://dx.doi.org/10.1016/j.jmatprotec.2017.05.040.

[15] Saunders FI, Wagoner RH. Forming of Tailor-Welded Blanks. Metallurgical and Materials Transactions. 1996;27(9):2605-2616. http://dx.doi.org/10.1007/BF02652354.

[16] Lee AP, Feltham E, Van Deventer J. Tailor welded blank technology for automotive applications. Sae Technical Paper Series. 1996;1:1-14. http://dx.doi.org/10.4271/960817.

[17] Zadpoor AA, Sinke J, Benedictus R, Pieters R. Mechanical properties and microstructure of friction stir welded tailor-made blanks. Materials Science and Engineering A. 2008;494(1-2):281-290. http://dx.doi.org/10.1016/j.msea.2008.04.042.

[18] Kim J, Kim S, Kim K, Jung W, Youn D, Lee J, et al. Effect of beam size in laser welding of ultra-thin stainless steel foils. Journal of Materials Processing Technology. 2016;233:125-134. http://dx.doi.org/10.1016/j.jmatprotec.2016.02.019.

[19] Shi MF, Pickett KM, Bhatt KK. Formability issues in the application of tailor welded blank sheets. Sae Technical Paper Series. 1993;1:1-11. http://dx.doi.org/10.4271/930278.

[20] Pallett RJ, Lark RJ. The use of tailor blanks in the manufacture of construction components. Journal of Materials Processing Technology. 2001;117(1-2):249-254. http://dx.doi.org/10.1016/S0924-0136(01)01124-4.

[21] Riahi M, Amini A, Sabbaghzadeh J, Torkamany MJ. Analysis of weld location effect and thickness ratio on formability of tailor welded blank. Science and Technology of Welding and Joining. 2012;17(4):282-287. http://dx.doi.org/10.1179/1362171812Y.0000000005.

[22] Companhia Siderúrgica Nacional. Galvanizados: descrição geral. São Paulo: CSN; 2019 [acesso em 1 maio 2019]. Disponível em: http://www.csn.com.br/conteudo_pti.asp?idioma=0\&conta=45\&tipo=59673

[23] Abbasi M, Ketabchi M, Labudde T, Prahl U, Bleck W. New attempt to wrinkling behavior analysis of tailor welded blanks during the deep drawing process. Materials \& Design. 2012;40:407-414. http://dx.doi.org/10.1016/j.matdes.2012.04.015.

[24] Ahmetoglu MA, Brouwers D, Shulkin L, Taupin L, Kinzel GL, Altan T. Deep drawing of round cups from tailor-welded blanks. Journal of Materials Processing Technology. 1995;53(3-4):684-694. http://dx.doi.org/10.1016/0924-0136(94)01767-U.

[25] Choi Y, Heo Y, Kim HY, Seo D. Investigations of weld-line movements for the deep drawing process of tailor welded blanks. Journal of Materials Processing Technology. 2000;108(1):1-7. http://dx.doi.org/10.1016/S0924-0136(00)00536-7.

[26] American Society for Testing and Materials. ASTM E8/E-8M: standard test methods for tension testing of metallic materials. 16th ed. West Conshohocken: ASTM; 2016. 30 p.

[27] Ghoo BY, Keum YT, Kim YS. Evaluation of the mechanical properties of welded metal in tailor steel sheet welded by $\mathrm{CO}_{2}$ laser. Journal of Materials Processing Technology. 2001;113(1-3):692-698. http://dx.doi.org/10.1016/S0924-0136(01)00674-4.

[28] Xu F, Sun G, Li G, Li Q. Experimental investigation on high strength steel (HSS) tailor-welded blanks (TWBs). Journal of Materials Processing Technology. 2014;214(4):925-935. http://dx.doi.org/10.1016/j.jmatprotec.2013.11.018.

[29] Kim D, Lee W, Kim J, Chung K-H, Kim C, Okamoto K, et al. Macro-performance evaluation of friction stir welded automotive tailorwelded blank sheets. Part II: formability. International Journal of Solids and Structures. 2010;47(7-8):1063-1081. http://dx.doi.org/10.1016/j.jijsolstr.2009.12.021.

[30] Callister WD, Rethwisch DG. Ciência e engenharia de materiais: uma introdução. 9. ed. São Paulo: LTC; 2016.912 p.

[31] Dieter GE. Mechanical metallurgy. 3rd ed. Boston: McGraw-Hill; 1988.

[32] Hosford WF, Caddell RM. Metal forming: mechanics and metallurgy. 3rd ed. Cambridge: Cambridge University Press; 2007.312 p. http://dx.doi.org/10.1017/CBO9780511811111. 
[33] American Society for Testing and Materials. ASTM E-92: standard test methods for vickers hardness and knoop hardness of metallic materials. 17th ed. West Conshohocken: ASTM; 2017. 27 p.

[34] American Society for Testing and Materials. ASTM E-384: standard test method for microindentation hardness of materials. 17th ed. West Conshohocken: ASTM; 2017. 40 p.

[35] Panda SK, Kumar DR, Kumar H, Nath AK. Characterization of tensile properties of tailor welded IF steel sheets and their formability in stretch forming. Journal of Materials Processing Technology. 2007;183(2-3):321-332. http://dx.doi.org/10.1016/j.jmatprotec.2006.10.035.

[36] Cheng $\mathrm{CH}$, Chan LC, Chow CL. Weldment properties evaluation and formability study of tailor-welded blanks of different thickness combinations and welding orientations. Journal of Materials Science. 2007;42(15):5982-5990. http://dx.doi.org/10.1007/s10853-0061126-0.

[37] American Society for Testing and Materials. ASTM E-3: standard guide for preparation of metallographic specimens. 11th ed. West Conshohocken: ASTM Internacional; 2017. 12 p.

[38] Colpaert H. Metalografia dos produtos siderúrgicos comuns. 4. ed. São Paulo: Blucher; 2008. 672 p.

[39] Gong H, Wang S, Knysh P, Korkolis YP. Experimental investigation of the mechanical response of laser-welded dissimilar blanks from advanced- and ultra-high-strength steels. Materials \& Design. 2016;90:1115-1123. http://dx.doi.org/10.1016/j.matdes.2015.11.057.

[40] Zhang H, Liu X, Liu L, Hu P, Wu J. Forming limit and thickness transition zone movement for tailor rolled blank during drawing process. Journal of Iron and Steel Research International. 2016;23(3):185-189. http://dx.doi.org/10.1016/S1006-706X(16)30032-2.

[41] Assunção E, Quintino L, Miranda R. Comparative study of laser welding in tailor blanks for the automotive industry. International Journal of Advanced Manufacturing Technology. 2010;49(1-4):123-131. http://dx.doi.org/10.1007/s00170-009-2385-0.

[42] Cetlin PR, Helman H. Fundamentos da conformação: mecânica dos metais. 2. ed. São Paulo: Artliber; 2005.265 p.

[43] Li Y, Lin J. Experimental and numerical investigations of constraint effect on deformation behavior of tailor-welded blanks. Journal of Materials Engineering and Performance. 2015;24(8):2957-2969. http://dx.doi.org/10.1007/s11665-015-1573-3.

[44] Narayanan RG, Naik BS. Assessing the validity of original and modified failure criteria to predict the forming limit of unwelded and tailor welded blanks with longitudinal weld. Materials and Manufacturing Processes. 2010;25(11):1351-1358. http://dx.doi.org/10.1080/10426914.2010.529588.

[45] Kinsey B, Liu Z, Cao J. A novel forming technology for tailor-welded blanks. Journal of Materials Processing Technology. 2000;99(13):145-153. http://dx.doi.org/10.1016/S0924-0136(99)00412-4.

[46] Safdarian R. The effects of strength ratio on the forming limit diagram of tailor-welded blanks. Ironmaking \& Steelmaking. 2018;45(1):17-24, 27. http://dx.doi.org/10.1080/03019233.2016.1235371.

[47] Dowling NE. Mechanical behavior of materials: engineering methods for deformation, fracture, and fatigue. 4th ed. London: Pearson Education; 2013. 977 p.

[48] Dharan CKH, Kang BS, Finnie I. Finnie's notes on fracture mechanics: fundamental and practical lessons. New York: Springer; 2016. Cleavage and ductile fracture mechanisms: the microstructural basis of fracture toughness; p. 201-213.

[49] Affonso LOA. Machinery failure analysis handbook: sustain your operations and maximize uptime. Houston: Gulf Publishing Company; 2007. Ductile and brittle fractures; p. 33-42.

[50] Safdarian Korouyeh R, Moslemi Naeini H, Torkamany MJ, Liaghat G. Experimental and theoretical investigation of thickness ratio effect on the formability of tailor welded blank. Optics \& Laser Technology. 2013;51:24-31. http://dx.doi.org/10.1016/j.optlastec.2013.02.016.

[51] Min KB, Kim KS, Kang SS. A study on resistance welding in steel sheets using a tailor-welded blank (1st report): evaluation of upset weldability and formability. Journal of Materials Processing Technology. 1998;2000(101):186-192.

[52] Krauss G. Steels: heat treatment and processing principles. 2nd ed. Ohio: ASM International; 1990. 497 p. 